\title{
Temporal changes in cardiac SPECT utilization and imaging findings: Where are we going and where have we been?
}

\author{
Milena J. Henzlova, $M D{ }^{a}$ and W. Lane Duvall, $M D^{b}$ \\ a Cardiology, Mount Sinai Health System, New York, NY \\ b Heart and Vascular Institute, Hartford Hospital, Hartford, CT
}

Received Mar 8, 2019; accepted Mar 8, 2019

doi:10.1007/s12350-019-01687-y

\section{See related article, 2167-2177}

SPECT myocardial perfusion imaging (MPI) has become the "grand dame" of cardiac imaging: it has come a long way from modest beginnings more than 50 years ago (planar Tl-201 imaging) to current CZT SPECT with CT attenuation correction and attempts for absolute coronary flow determination. It has survived continuous predictions of imminent demise due to perceived danger of internal radiation and from the advent of new imaging modalities: stress echocardiography, positron emission tomography, and coronary CT angiography. The robust diagnostic and prognostic power of SPECT MPI has kept it relevant for decades with recent reports of decreasing volume and rate of abnormal findings again sounding the alarm of its demise.

To separate myth, wishful thinking and personal preferences from reality, we can now turn to real data.

With the publication in this issue of the Journal of Nuclear Cardiology international data from Italy can now be added to the large amount of data defining trends of utilization of SPECT cardiac imaging and distribution of abnormal findings in the past 25 years. ${ }^{1}$ Most available data originated in the USA, by far the largest per capita utilizer of diagnostic cardiac imaging in the world. Five tertiary medical centers' databases have been interrogated, and the results were published between 2013 and 2019. Data is available from the West

\footnotetext{
Reprint requests: W. Lane Duvall, Hartford Hospital, Hartford, CT; Lane.Duvall@hhchealth.org

J Nucl Cardiol 2020;27:2178-82.

$1071-3581 / \$ 34.00$

Copyright (c) 2019 American Society of Nuclear Cardiology.
}

Coast (Rozanski et al 2013, Los Angeles, CA), ${ }^{2}$ East Coast (Duvall et al 2015, New York City and Hartford, CT), ${ }^{3}$ from the Midwest (Thompson, 2015, Kansas City, MO and Jouni et al 2016 and 2017, Rochester, MN) ${ }^{4-6}$ and now from Italy (Megna et al 2019, Naples, Italy). ${ }^{1}$ Covered years span from 1991 to 2017 and include data from over 352,000 patients.

Rozanski et al analyzed data from first MPI studies of 39,515 patients without known coronary artery disease (CAD) performed at Cedars-Sinai Medical Center in Los Angeles, CA from January 1991 till December 2009. ${ }^{2}$ Patients with known cardiomyopathies and valve disease were excluded. This study also incorporated follow-up including all-cause mortality using the Social Security Death Index (mean follow up of $9.3 \pm 4.7$ years).

The total annual volume of tests increased between 1991 and 2005, then decreased during the last quartile (2006-2009). During the observed 18 years some demographic factors changed in favor of decreasing CAD risk factors: patient age decreased, the proportion of female patients increased, and smoking decreased while other demographic factors changed to increase the burden of CAD risk factors as BMI increased and the prevalence of hypertension, hyperlipidemia and diabetes increased. During this time period the percentage of abnormal studies decreased from $40.9 \%$ in 1991 to $8.7 \%$ in 2009 (i.e., $91.3 \%$ normal studies!) and the prevalence of ischemic SPECT MPI studies decreased from $29.6 \%$ in 1991 to $5.0 \%$ in 2009 while annual mortality rates decreased slightly from $3.3 \%$ to $2.6 \%$. The proposed explanations for these dramatic changes include improved modifiable risk factor management and changes in referral patterns to stress SPECT MPI such as more frequent direct referral to catheterization of more symptomatic patients.

Duvall et al analyzed data from combined databases of Mount Sinai Medical Center in New York, NY and 
Harford Hospital in Harford, CT. ${ }^{3}$ All patients undergoing their first SPECT or PET MPI between January 1996 and December 2012 were included: 63,065 with no known CAD and 45,589 with known CAD (a total of 108,654 patients). Invasive coronary angiography results (obstructive vs. nonobstructive CAD) from the same time interval were also separately reviewed.

The annual volume of MPI studies increased between 1996 and 2005 and then decreased in the final time period from 2009-2012. The proportion of patients being tested with known CAD decreased during the observed period (from $53.4 \%$ to $32.0 \%$ ). In regards to the cardiac risk factor burden, the mean patient age did not change, the proportion of female patients increased, BMI increased as did the prevalence of hypertension, hyperlipidemia and diabetes, and while smoking initially increased and then decreased in the last quartile it remained more prevalent in the last time period than the first. During this 17 year period the percentage of normal studies increased in all patients from $46.2 \%$ to $68.2 \%$, both in patients without known CAD (from $67.8 \%$ to $82.0 \%$ ) and with known CAD (from $25.3 \%$ to $37.9 \%$ ). Coronary angiograms $(\mathrm{N}=142,924)$ done during the observed period (on allcomers, not only patients with MPI) showed a decrease in obstructive CAD (from $77.2 \%$ to $67.9 \%$ ), with a corresponding decrease of left main and three vessel disease diagnosis. There was also an increase in the finding of non-obstructive CAD (from $11.8 \%$ to $17.6 \%$ ). Proposed explanations for the changes included better modifiable risk factor management, increased direct referral of sicker patients to coronary angiography and possible fewer false-positive MPI studies due to technical advances.

Thompson reviewed the experience at St. Luke's Mid America Heart Institute in Kansas City between 2002 and 2014. ${ }^{4}$ He briefly reported on the frequency of ischemic MPI studies in over 140,000 patients. This data showed that the prevalence of ischemic studies decreased from a peak of approximately $45 \%$ in 2003 to approximately $22 \%$ in 2012 , only to increase slightly in the last two years of assessment to approximately $28 \%$.

Jouni et al analyzed data from the Mayo Clinic, Rochester, Minnesota (two published studies in 2016 and 2017). ${ }^{5,6}$ Data were collected between January 1991 and December 2012. Patients with a left bundle branch block, paced rhythm, known cardiomyopathies and valve disease were excluded. Results of the first SPECT study done in patients without known CAD ( $\mathrm{N}=35,894$ patients) and with known CAD ( $\mathrm{N}=19,373$ patients) were analyzed separately. The volume of stress echocardiography during this period was also reviewed.

The annual volume of SPECT MPI tests increased from 1991 until the middle of the observed period (2003-2004) and then declined. Parallel utilization of stress echocardiography and treadmill exercise testing revealed a similar pattern, thus no clear evidence of test substitution as a cause of the SPECT MPI volume or result findings. Cardiac risk factor changes occurred in both groups (no known and known CAD), with increasing risk factor burden as patient age increased, the proportion of male patients increased, and the prevalence of hypertension and hyperlipidemia increased, and with decreasing risk factor burden as smoking decreased, BMI remained unchanged, and diabetes increased in patients with known CAD only. The percentage of low risk SPECT studies (SSS $=0-3$ ) increased from $63.9 \%$ to $95.6 \%$ (!) in patients with no known CAD and from $27.8 \%$ to $77.1 \%$ in patients with known CAD, with a similar decrease in severely abnormal studies (SSS $\geq 9$ ) (from $16.1 \%$ to $2.2 \%$, and from $47.8 \%$ to $14.8 \%$, respectively). As an explanation for the decline in SPECT MPI volume, the authors point to the use of Appropriateness criteria and decreases in study reimbursement. They attribute the decrease in perfusion abnormalities to better modifiable risk factors management, effect of mechanical revascularization and direct referral of sicker patients to coronary angiography. More patients with a history of PCI or CABG had low risk scans at the end of the observed period, compared to the beginning ( $40.5 \%$ and $89.4 \%$, respectively).

Megna et al from Naples, Italy in this issue of the Journal presents the first European cardiac SPECT utilization and results data, covering the time period from January 2006 to December 2017. Included were all patients $(\mathrm{N}=8,886)$ both with and without known CAD. The volume of performed studies continued to increase over this time period. In regards to the burden of cardiac risk factors, patient age increased, the prevalence of hypertension and hyperlipidemia increased, and smoking increased from $28 \%$ to $47 \%$ (!) while the proportion of female patients increased and diabetes remained unchanged. The percentage of normal studies changed very little over this time period. In patients with no known CAD normal studies increased from $80 \%$ to $82 \%$, and in patients with known CAD (42\% of total population) from $36 \%$ to $38 \%$.

Despite both the geographic and temporal differences, the results are consistent in the US: the total volume of performed studies decreased, the number of traditional risk factors increased, and the prevalence of abnormal and severely abnormal studies decreased. We now know that similar, but less pronounced trends occurred in Italy.

\section{VOLUME}

In all of the reviewed US tertiary centers, the volume of first-time cardiac SPECT studies increased from the 1991-1996 baseline until 2010 and 2013, with 
limited growth at the end of the observed periods. From published Medicare data, cardiac imaging increased between 2001 and 2006 from 63.4 to 88.0 studies per 1,000 beneficiaries and decreased to 61.9 per 1000 in 2013. ${ }^{7}$ Based on available Medicare and Medicaid Part $\mathrm{B}$ data, we compiled the volume of non-invasive studies performed for the diagnosis of CAD from 2000-2017 including all SPECT, PET, stress echocardiography, and coronary CTA (Figure 1). ${ }^{8}$ SPECT volume increased to a peak during 2005 and 2006 and then declined from there leveling off in 2016-2017. To provide some perspective, SPECT annual volume was several orders of magnitude greater than stress echocardiography which peaked in 2004, and many times the volume of coronary CTA or PET, whose volume surpassed that of CTA in 2009-2010.

The dramatic decrease in number of cardiac SPECT studies performed in the USA over the observed periods temporally coincides with the wide spread closures or mergers of private cardiology offices with local hospitals. This trend coincided with and was driven in part by declining study reimbursement, which likely had the effect of eliminating unhealthy incentives for poorly indicated first, as well as repeat MPI studies. The introduction of mandatory test pre-approvals by insurance companies while possibly limiting access to some appropriate testing, likely eliminated many non-indicated studies. The introduction of Appropriate use criteria also contributed to the elimination of some unnecessary and repetitive studies, further decreasing study volume.

Test substitution for another non-invasive modality would appear to be a less likely explanation for SPECT volume changes. This can be seen in the lack of a substantial increase in stress echocardiography volume,$^{5-7}$ or CTA volume increase during the observed periods. $^{7}$ Levine et al reports that in 2013 the ratio of SPECT MPI to CTA was 58:1 and only $3.9 \%$ of all Medicare MPI studies utilized PET. ${ }^{7}$ These two more recent imaging modalities hold a similarly small proportion of the non-invasive imaging market and the availability, cost and expertise remain limited and out of reach for most of outpatient offices.

Finally, direct referral to the catherization laboratory given the proven benefits of early mechanical interventions for acute coronary syndromes and improved safety profile of radial access may explain the lower utilization of pre-catheterization non-invasive testing. This phenomenon of direct catheterization referral may be implied from the declining number of patients with typical angina referred for SPECT MPI as these more symptomatic patients may have bypassed non-invasive testing altogether.

This loss of MPI volume has not been seen in the non-US experience. Published SPECT MPI volume data from Germany reports an increase in cardiac SPECT from 2009 to 2015. ${ }^{9}$ Similarly, SPECT utilization increased in Australia from 287 to 337 studies per 100,000 people in 11 years. $^{10}$ The current study from Italy reports uninterrupted growth between 2006 and 2017. Clearly, factors other than those discussed above are at play overseas as different healthcare delivery systems, local regulations, traditions, equipment and tracer availability have an important influence on study volume.

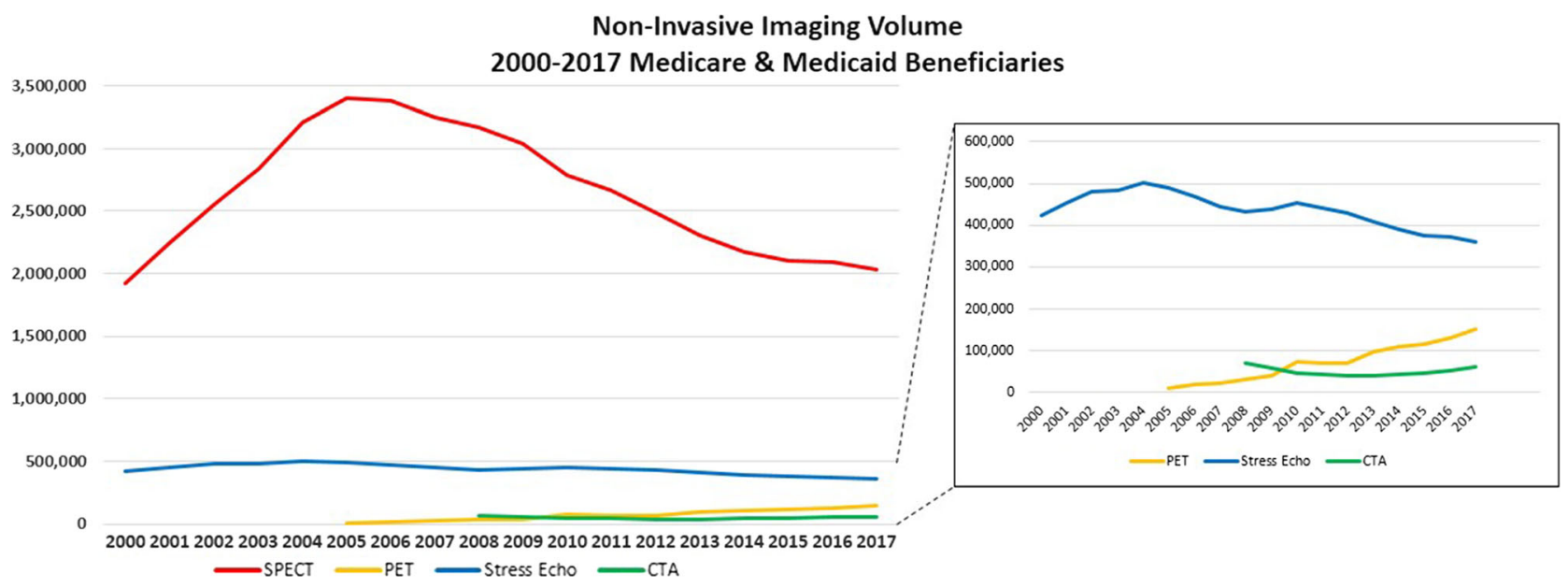

Figure 1. Medicare and Medicaid data from the Part B National Summary Data File on noninvasive imaging studies for the diagnosis of $\mathrm{CAD}{ }^{8}$. 


\section{RISK FACTORS AND PRE-TEST STRATIFICATION}

The change in prevalence of traditional risk factors is consistent in all reviewed studies: while patient mean age did not change significantly, the prevalence of hypertension, hyperlipidemia and diabetes increased; smoking decreased in the US (but increased in Italy). Prevalence of patients with known versus no known CAD declined in the US cohorts, but increased in Italy. While it is possible, that the detection of cardiac risk factors such as diabetes, hypertension and hyperlipidemia improved over time as a result of greater efforts at screening without a real change in prevalence (early detection bias), the well-known and documented obesity epidemic, has undoubtedly contributed to an increase in diabetes.

Recent studies have shown that both short and longterm risk prognosis is better than expected when derived from simple scores based on traditional risk factors only. ${ }^{11}$ It would seem that over the past decade, medical therapy (including statins, antiplatelet agents, hypertension management) and revascularization therapy (PCI/ CABG) along with lifestyle modifications (smoking cessation and exercise) have changed the natural history of atherosclerosis. This is born out in the studies on the temporal trends of SPECT results, on all-cause mortality decrease, ${ }^{2}$ on diminished severity of CAD detected by coronary angiography, ${ }^{3}$ and on the increased proportion of normal studies in revascularized patients. ${ }^{6}$

It is obvious, that pre-test risk stratification based only on the number of traditional risk factors is no longer adequate. We would expect the best adherence to Appropriate use criteria in the tertiary medical centers reporting their results. And still, $91 \%$ of studies to "rule out" CAD were normal in Los Angeles, $82 \%$ in NY and CT, and $96 \%$ in Rochester, MN! There is a need for the development of new pre-test assessment algorithms: Does-treated hypertension or hyperlipidemia pose the same risk as untreated hypertension or hyperlipidemia? Is former smoking as important a risk factor as current smoking? Is it appropriate to combine type I and type II diabetes mellitus in the same risk category? How does complete versus incomplete revascularization alter the natural history of CAD? Novel risk classification would in many patients downgrade the pre-test risk and negate the need for testing. We have already begun to see the beginning of coronary artery calcium scoring emerging as a quick and cost-effective "re-classifier." 12

\section{ABNORMAL FINDINGS}

The main message from the US data is that despite the higher prevalence of traditional risk factors, the prevalence of abnormal (and severely abnormal) SPECT

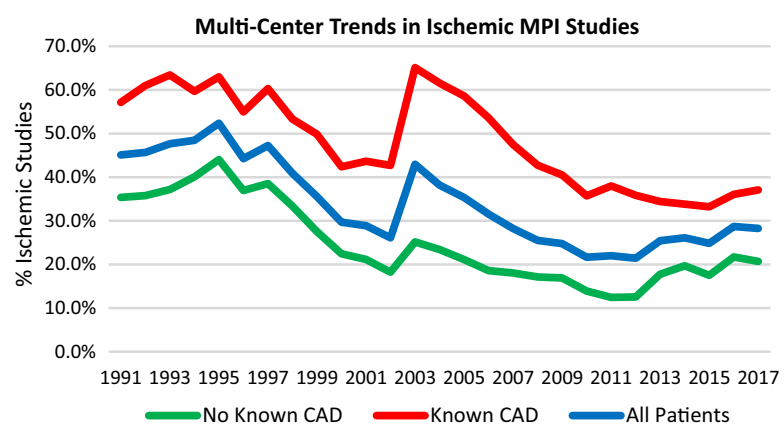

Figure 2. Composite data from St. Luke's Mid America Heart Institute, Kansas City, the Mayo Clinic, Rochester, Mount Sinai, New York, and Hartford Hospital, Hartford expressing the percent of ischemic MPI studies in 245,000 patients from 1991 to 2017.

results decreased in all observed cohorts (but not in Europe). The data on ischemic MPI studies from 3 of the 4 institutions who have published their data can be seen in Figure 2. SPECT technology has surely evolved over the past decades although the impact of these advancements on the rate of abnormal studies is not clear. Improvement in diagnostic accuracy was achieved with the transition from Tl-201 to Tc-99m tracers and with introduction of image gating. However, this transition was mostly competed in the early 1990s, prior to the time period of documented declining perfusion abnormalities. Technological advancements with dual head cameras, iterative reconstruction, and high-efficiency CZT SPECT cameras may have reduced imaging artifact from low-count studies or motion artifact from prolonged imaging, thereby improving specificity and reducing false positives. Utilization of attenuation correction promised to eliminate most false-positive findings from attenuation artifacts (breast and diaphragmatic attenuation). This promise of new technology still has not been fully realized since additional hardware (CT or transmission sources for attenuation correction) was not adopted by most laboratories due to additional cost and lack of reimbursement, and other new technology adoption was held back by the slow turnover of older cameras for ones with new technology.

The majority of larger medical centers have access to a multitude of invasive and non-invasive cardiac imaging modalities. Obviously, the concept of "onestop shopping" did not materialize. Nuclear imaging (SPECT/PET), stress echocardiography or CTA, all appear to be an appropriate first test for many patients. Test substitution with the distribution of certain patient types to other imaging modalities may have resulted in the findings seen in these studies. However, analyzing the results of one modality only (MPI studies), does not allow for an overview of the changing referral patterns 
and imaging results despite similar demographics (age, gender). So far, only stress MPI and invasive coronary angiography have presented temporal trends in cardiac imaging results. ${ }^{13,14}$ Similar data from stress echocardiography and coronary CTA is missing and would allow for a better understanding of trends across all noninvasive modalities.

If the trend of fewer abnormal or severely abnormal findings is consistent across different imaging modalities, the most likely explanation would remain that there has been a favorably changed substrate of epicardial CAD due to current medical therapy, lifestyle modification, and revascularization interventions. If true, then both referrals for the detection and risk stratification of epicardial CAD need to be adjusted to the new landscape for the benefit of individual patients as well for the good of healthcare resources utilization.

\section{Acknowledgements}

We would like to thank Drs. Thompson and Al Badarin from St. Luke's, and Drs Gibbons and Jouni from the Mayo Clinic for providing their data on ischemic MPI studies from their institutions.

\section{Disclosure}

Milena J. Henzlova and W. Lane Duvall indicated that they have no financial conflict of interest.

\section{References}

1. Megna R, Zampella E, Assante R, Nappi C, Gaudieri V, Mannarino $\mathrm{T}$ et al. Temporal trends of abnormal myocardial perfusion imaging in a cohort of Italian subjects: relation with cardiovascular risk factors. J Nucl Cardiol 2019.

2. Rozanski A, Gransar H, Hayes SW, Min J, Friedman JD, Thomson LE, et al. Temporal trends in the frequency of inducible myocardial ischemia during cardiac stress testing: 1991 to 2009. J Am Coll Cardiol 2013;61:1054-65.

3. Duvall WL, Rai M, Ahlberg AW, O'Sullivan DM, Henzlova MJ. A multi-center assessment of the temporal trends in myocardial perfusion imaging. J Nucl Cardiol 2015;22:539-51.
4. Thompson RC, Allam AH. More risk factors, less ischemia, and the relevance of MPI testing. J Nucl Cardiol 2015;22:552-4.

5. Jouni H, Askew JW, Crusan DJ, Miller TD, Gibbons RJ. Temporal trends of single-photon emission computed tomography myocardial perfusion imaging in patients without prior coronary artery disease: A 22-year experience at a tertiary academic medical center. Am Heart J 2016;176:127-33.

6. Jouni H, Askew JW, Crusan DJ, Miller TD, Gibbons RJ. Temporal Trends of Single-Photon Emission Computed Tomography Myocardial Perfusion Imaging in Patients With Coronary Artery Disease: A 22-Year Experience From a Tertiary Academic Medical Center. Circ Cardiovasc Imaging 2017;10.

7. Levin DC, Parker L, Halpern EJ, Rao VM. Recent Trends in Imaging for Suspected Coronary Artery Disease: What Is the Best Approach? J Am Coll Radiol 2016;13:381-6.

8. Centers for Medicare and Medicaid Services. Part B National Summary Data File. https://www.cms.gov/research-statistics-dat a-and-systems/downloadable-public-use-files/part-b-national-sum mary-data-file/overview.html.

9. Hellwig D, Marienhagen J, Menhart K. Nuclear Medicine in Germany. Updated key data and trends from official statistics. Grosse J Nuklearmedizin 2017;56:55-68.

10. Fonseca R, Otahal P, Wiggins N, Marwick TH. Growth and geographical variation in the use of cardiac imaging in Australia. Intern Med J 2015;45:1115-27.

11. Cheng VY, Berman DS, Rozanski A, Dunning AM, Achenbach S, Al-Mallah M, et al. Performance of the traditional age, sex, and angina typicality-based approach for estimating pretest probability of angiographically significant coronary artery disease in patients undergoing coronary computed tomographic angiography: Results from the multinational coronary $\mathrm{CT}$ angiography evaluation for clinical outcomes: an international multicenter registry (CONFIRM). Circulation 2011;124:1-8.

12. Berman DS, Rozanski A. Value-based imaging: Combining coronary artery calcium with myocardial perfusion imaging. J Nucl Cardiol 2016;23:939-41.

13. Patel MR, Peterson ED, Dai D, Brennan JM, Redberg RF, Anderson HV, et al. Low diagnostic yield of elective coronary angiography. New Engl J Med 2010;362:886-95.

14. Ko DT, Tu JV, Austin PC, Wijeysundera HC, Samadashvili Z, Guo H, et al. Prevalence and extent of obstructive coronary artery disease among patients undergoing elective coronary catheterization in New York State and Ontario. JAMA 2013;310:163-9.

Publisher's Note Springer Nature remains neutral with regard to jurisdictional claims in published maps and institutional affiliations. 